\title{
PROTOCOLO DE SISTEMAS HOGAR EUROPEO: CONCEPTOS Y PRODUCTOS
}

\author{
(THE EUROPEAN HOME SYSTEMS PROTOCOL: CONCEPTS AND PRODUCTS)
}

\author{
Pierre Guillemin \\ SGS-THOMSON Microelectronics
}

ESPAÑA
Fecha de recepción:10-XII-98

106-7

\section{RESUMEN}

El ahorro en el gasto de energía, la seguridad de personas y bienes, el aumento/mejora del bienestar son algunas de las ventajas que procura la Automatización del Hogar. El ahorro de energía se consigue gracias a la planificación de la temperatura según el número y tipo de habitaciones, adaptación automática del consumo al coeficiente de precio y utilización de alumbrado. La seguridad de personas y bienes se incrementa gracias a funciones tales como detectores de incendios o escapes de gas, alarma telefónica o detector antirrobo. Un incremento de la calidad de vida conlleva nuevos recursos para nuestros mayores, con la distribución a domicilio de pasatiempos y servicios y el control remoto telefónico de calefacción y alumbrado. Para alograr estos nuevos servicios, los equipos de hogar, conectados a los diferentes medios de comunicación, pueden relacionarse para intercambiar mensajes de forma estructurada según unas normas de comunicación. Este es el objetivo del protocolo de Sistemas de Hogar Europeo.

Nacido en 1992 con la publicación de una especificación bien definida de un protocolo de comunicación abierta, dedicado a la Automatización del Hogar, la European Home Systems (EHS) ha entrado ya en su fase de madurez: existen productos hardware y software y las compañias europeas más importantes implicadas en bienes muebles y servicios han decidido incluir el protocolo EHS en sus productos. Este articulo describe el ambiente en el que se ha desarrollado el EHS y enumera los diferentes requisitos que conducirán al los conceptos del PLUG \& PLAY (Conecta y Juega). Asi pues, se exponen, brevemente, los componentes software y hardware disponibles para desarrollar la aplicación del EHS.

\section{SUMMARY}

Energy cost savings, security of persons and goods, comfort improvement are some of the benefits of Home Automation. Energy cost savings are achieved thanks to temperature management depending on presence and type of rooms, automatic adaptation of power consumption to tariff rateslighting management. Security of persons and goods is increased with functions such as detection of fire of gas leakage, transmission by telephone of alarm or detection of intrusion. Increasing of quality of life brings new facilities for elderly, in-house distribution of entertainment and services, remote control by telephone of heating and lighting. To achieve all these new services, home appliances, connected on different communication media, have to communicate to exchange messages in a structured way following a communication standard. This is the purpose of the European Home Systems protocol.

Born in 1992 by the publishing of a comprehensive specification for an open communication protocoldedicated to Home Automation, European Home Systems (EHS) has now entered a mature phase: hardware and software products exist and major European companies involved in white and brown goods and major European utilities have decided to include the EHS protocol in their products. This article describes the environment in which EHS has been developed, lists the different requirements issued from market studies for home automation. It explains the technical concepts answering these requirements leading to the PLUG \& PLAY concepts. Then, this article briefly describes the software and hardware components available to develop an EHS application. 


\section{DESARROLLO DEL AMBIENTE EHS}

Desde 1984 la Comisión Europea ha patrocinado varios proyectos destinados al Home Systems.

En estos proyectos incipientes figuran implicados los principales proveedores europeos de productos White and Brown, así como las compañías de electricidad y telecomunicación y fabricantes de equipos y sistemas europeos. Esta participación garantiza el desarrollo de un protocolo abierto, con amplio espectro de aplicación, que permita que equipos procedentes de distintos fabricantes puedan comunicarse entre sí. Los proyectos se han definido en las especificaciones del European Home System: un documento -claro y conciso- publicado en 1992, que constituye una base para el desarrollo de los productos de automación del hogar.

Desde 1992 hasta finales del 1995, los proyectos de Componentes-EHS se dedican a la divulgación de productos hardware y software basados en las especificaciones EHS. Los resultados incluyen la línea de fuerza Modem SGS-THOMSON ST7537HS1, el prototipo interfase Twisted Pair 1, el software de comunicación, disponible en microcontrolador ST9 y componentes relacionados, e instrumentos de desarrollo software.

Entretanto, varios proyectos están desarrollando pruebas experimentales de productos y sistemas. Por ejemplo, el proyecto HS-TCTS ha diseñado un mecanismo para llevar a cabo las pruebas de conformidad de los productos EHS. Esta herramienta de trabajo se encuentra ya a disposición en ACERLI (1) y en el Instituto FRAUNHOFER (2). Los proyectos GAP y ELECline se disponen a desarrollar productos EHS dedicados a Gas y Electricidad. Momentum y Horbit proyecta utilizar y eventualmente ampliar las especificaciones EHS para la automatización de edificios.

La Asociación Europea de Sistemas de Hogar (European Home Systems Association, EHSA) es una organización abierta, pensada para mantener y divulgar la especificación EHS. Dentro de EHS, el Comité para el Control de las Normas (Standard Control Committee (SCC)) es el artífice del fortalecimiento de la especificación EHS y de la coordinación de las actividades del Grupo InterOperabilidad (Inter-Operability Group, IOG) que controla la inter-operabilidad entre equipos, a nivel de aplicación.

\section{ESPECIFICACIÓN Y ARQUITECTURA DEL EHS}

Una definición de la especificación EHS hace que todos los equipos electrodomésticos puedan comunicarse entre sí y compartir prestaciones. El protocolo EHS se basa en la comunicación compartida de sistemas y en definiciones claras de la funcionalidad del aparato.

La especificación hace una descripción completa de todos los aspectos de la comunicación. El modelo de comunicación EHS se basa en el modelo de referencia de la estructura de Normas de Intercomunicación Abierta (Open Standard Intercommunication (OSI)). El EHS especifica la capa/nivel física/o, la capa/nivel de conexión de datos, la capa/de red y la capa/de aplicación. Debido a que la longitud del mensaje es limitada, la sesión de diálogo es corta y el lenguaje de los mandos queda controlado

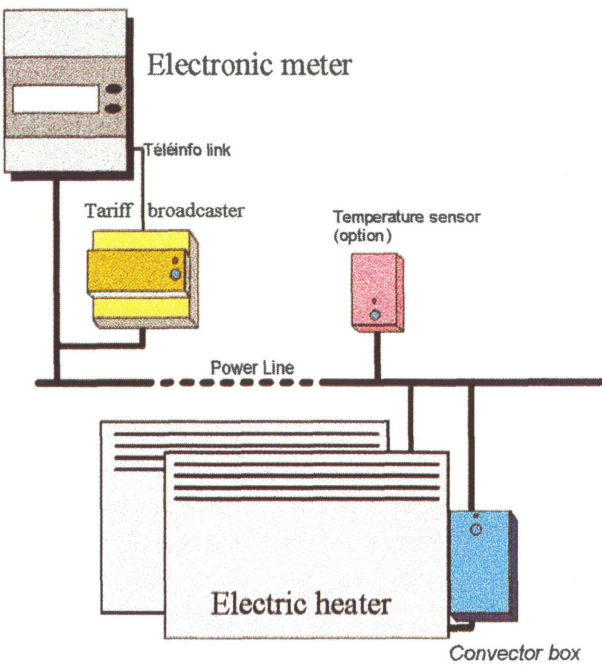

Living zone

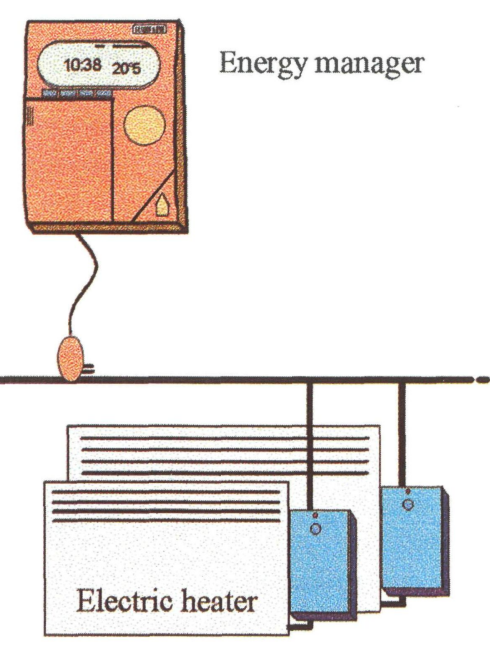

Rest zone

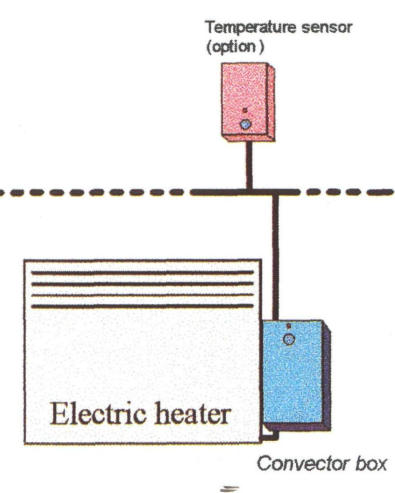

High comfort zame

Figura 1.- Ejemplo de aplicación de la arquitectura EHS. 
por la capa de aplicación; se ha omitido la capa de transporte, sesión y presentación.

La capa de aplicación traduce el lenguaje de aplicación a una estructura de datos capaz de circular por la red. Las Capas de Conexión de Datos (Data Link Layers), divididas en dos sub-capas Control de Acceso Medio (Médium Acceso Control, MAC) y Control de Conexión Lógico (Logical Link Control (LLC)) operan sobre la conversión de la corriente de bites, la reglamentación de acceso a la red, la identificación de estructuras, aportando un mecanismo de reconocimiento y repetición. La capa de red es idéntica para todas las unidades EHS y gestiona los datos relacionados con las rutas (por ejemplo, la dirección para llegar a una unidad pasando por varias sub-capas).

La especificación describe en su totalidad varias capas fisicas. Varias capas físicas han sido ya definidas teniendo en cuenta la variedad de requisitos de aplicación. Se pueden usar las capas físicas de Línea de Fuerza (Oiwer Line, PL), Infra Rojos (Infra Red, IR) o Frecuencia de Radio (Radio Frequency, RF) en un hogar para una comunicación a baja velocidad sin coste de sistema de cables. Cuando se necesita una velocidad más alta o transmitir señales análogas se puede usar un conductor doble trenzado (Twisted Pair, TP) o un cable coaxil. Por ejemplo, la comunicación por línea de fuerza eléctrica es apta para la comunicación entre el sistema de gestión de energía y los calentadores eléctricos. En contraposición, la comunicación por conductor doble trenzado es mejor en cuanto a sistema de seguridad.

La especificación está abierta a una evolución futura. Existe la posibilidad de introducir nuevas capas físicas y nuevos dominios de aplicación. La evolución de la especificación hacia la Automatización de la Casa está en marcha (proyecto Momentum y Horbit). Una mejora reciente de la especificación es la integración de un nuevo medio de conductor doble trenzado llamado TPO idéntico a la capa física BatiBUS.

La especificación describe las prestaciones "PLUG \& $P L A Y$ ". En un principio se identificaron algunos requisitos clave del mercado para el sistema de automatización del hogar. Estos requisitos clave para el mercado han sido incluidos en la especificación EHS y aportan un paquete de herramientas a nivel de aplicación que pueden ser útiles a la hora de diseñar un sistema "Plug \& Play". Diseñar un sistema de automatización de hogar "Plug \& Play" con EHS proporciona al usuario las ventajas siguientes:

Inter-operabilidad. Crear y difundir la necesidad de un sistema de comunicación es fundamental para la Automatización del Hogar. Ello implica que los electrodomésticos/aparatos que fabrican empresas independientes puedan funcionar en interdependencia/ cooperación. Así pues, la ventaja directa para el usuario es que no tiene más que elegir la marca que le guste.
Expandibilidad, Adaptabilidad, Configuración automática . En un Sistema de Automatización del Hogar, se pueden colocar los aparatos en cualquier punto de la red y se les puede cambiar de sitio con facilidad. Se puede configurar la red de forma automática (Plug \& Play) o manual, para respetar las diferentes zonas del hogar. Por ejemplo, un sistema de control de calefacción tiene que tener en cuenta la intensidad de calor que necesita cada zona (cuarto de estar, dormitorio, etc.).

Un Sistema de Automatización del Hogar basado en EHS es fácilmente expandible. Se puede añadir a la red nuevas aplicaciones/usos y nuevos niveles fisicos en cualquier momento. Esta funcionalidad modular ofrece al usuario la posibilidad de empezar con lo más elemental del aparato y añadirle prestaciones, según convenga.

Inter-conexión de las aplicaciones/funciones. Dentro de la misma red las aplicaciones de distinta procedencia pueden comunicar sin interferir unas con otras. Por ejemplo, un sistema de gestión de energía ni altera ni interrumpe el sistema de seguridad. Units/aparatos también pueden utilizar las prestaciones de otras units.

\section{CONCEPTOS TÉCNICOS DEL EHS: UNA RESPUESTA AL SISTEMA DE DISEÑO PLUG \& PLAY}

\subsection{Inter-operabilidad}

La arquitectura de la red EHS se basa en la noción de controladores y dispositivos compartidos dentro de los dominios de aplicación.

El controlador de características (feature controller, FC) controla la aplicación y configura los rasgos y la inteligencia de la aplicación, tales como recursos de gestión, algoritmos de control o de procesos de toma de decisiones. Un controlador define un dominio de aplicación, pero puede cubrir varios dominios de aplicación compartiendo sus recursos/posibilidades.

Un dispositivo regula y controla la aplicación de recursos. Por ejemplo, un calefactor eléctrico proporciona la fuente de calor, un termostato controla el nivel de temperatura. Cada dispositivo pertenece a un dominio de aplicación, pero puede estar compartido o controlado por varios controladores. Cada dispositivo/aparato está descrito por un Dispositivo de Descripción (Device Descriptor (DD)) codificado por la especificación. Así, aparatos/dispositivos que tengan un mismo DD son intercambiables. Este DD de dos bytes/bitios proporciona la información necesaria de tal forma que un controlador sabe qué fuentes estan libres/available en la red y cómo alcanzar esas fuentes. El primer byte/bitio es el dominio de aplicacién, el segundo byte proporciona la descripción del p*opio aparato $(\mathrm{DD}=1611$ representa un sensor de temperatura ambiente 
en el dominio de aplicación de calor). Controladores y dispositivos/aparatos pueden establecer una conexión lógica entre sí, llamada incorporación, para definir una aplicación de dominio. Un aparato que pueda ser controlado por un controlador se llama complex device (CoD).

Elementos de control de aplicación y elementos fuente pueden actuar recíprocamente a través de órdenes de ejecución. Se ha especificado un lenguaje de órdenes basado en la definición de objetos normalizados y de servicios asociados que regula dichos objetos. La estructura básica de una orden de ejecución es: dominio de aplicación, objeto, servicio, parámetros.

\subsection{Expandibilidad, flexibilidad en la colocación, configuración automática}

\subsubsection{Estructura de la red}

El EHS especifica varios niveles físicos. La estructura de la red puede estar formada por diferentes sub-redes que se basan en estos niveles físicos. Se puede formar una asociación de hasta siete capas/niveles físicos diferentes. Las rutas permiten la comunicación por las sub-redes EHS. Las puertas de entrada permiten la inter-conexión de la red EHS con una red que no sea EHS.

\subsubsection{Dirección de los elementos}

Cada elemento conectado a una sub-red tiene su propia dirección. La expandibilidad de la red queda garantizada, ya que un elemento conectado a una sub-red puede comunicarse con otros elementos situados en otras subredes. Esto se consigue a través de un esquema de direcciones normalizado para cada elemento. La unidad de direcciones consiste en la dirección sub-red del elemento de destino, el número de rutas/vías y las direcciones de los diferentes planos que tienen como destino la sub-red.

\subsubsection{Procedimiento de registro}

La función de gestión de la red proporciona el procedimiento de registro y éste se produce en el momento de la instalación (categoría I de registro) o cada vez que se conmuta/conecta el sistema (categoría II de registro). Durante este proceso, un elemento recién conectado negocia la dirección a través de un elemento específico llamado Controlador Medio (Medium Controller (MdC)), responsable de designar la dirección a una sub-red. El Controlador Medio es opcional y su funcionalidad puede quedar incluida dentro del controlador de características. Cuando no hay MdC en la sub-red, se hace el registro a través de un mecanismo de Asignación de Direcciones Distribuidas (Distributed Address Assignment(DAA)). En este caso, el elemento escoge al azar una dirección y envía un mensaje a esa dirección. Si no hay respuesta a este mensaje, la unidad/elemento mantiene la dirección. Si recibe contestación, la unidad/elemento escoge una dirección nueva y repite el proceso anterior.

\subsubsection{Procedimiento de captación}

Mantener una configuración automática y tener acceso a cualquier modificación de la configuración de la red, de los controladores y de los elementos que pertenecen a los mismos dominios de aplicación favorece la creación de interconexiones. La creación de estas conexiones se llama "enrolment"/captación/incorporación. Es éste un procedimiento por el que el controlador localiza a posibles participantes en la comunicación, recoge información sobre sus recursos de aplicación dentro del sistema y establece lazos con aquellos participantes en los que el controlador está interesado.

El proceso de captación/incorporación está basado en el intercambio de DD-Device Descriptors- entre controlador y componentes y la actualización de las tablas su Directorio(Aplication Title Directory (ATD)). El ATD, gestionado por el nivel/capa de aplicación de cada elemento/unidad, contiene el listado de direcciones y toda la información de red de las correspondientes unidades/elementos que la aplicación reconoce. Por ejemplo, un controlador de calefacción incorporará calefactores y sensores de temperatura para crear un dominio de aplicación de calor, perteneciente a una aplicación audio-vídeo.

\subsubsection{Estructura del marco EHS y corrección de errores}

El marco está compuesto por diferentes niveles/capas desde el nivel/capa de aplicación hasta el nivel/capa de red. Así, el nivel/capa de conexión de datos reserva el marco para el nivel/capa físico. En la línea de energía eléctrica, el marco EHS está compuesto por varios campos/zonas. Algunas se ocupan principalmente de la transmisión de energía. El preámbulo se utiliza para activar el módem de recepción. Durante la recepción de este campo/zona, el módem receptor comprueba si la señal recibida es un EHS que se conforma a la señal FSK y se sincroniza con esta señal.

En realidad el superior es quien marca la puesta en marcha de datos útiles. No se le puede confundir con el preámbulo. El receptor lo tiene en cuenta siempre que la diferencia entre el valor recibido y el esperado no exceda de 2 bites. La dirección de la casa se utiliza para desechar marcos procedentes de otras casas. Los dos últimos bytes del marco EHS contienen la Secuencia de Comprobación de Marco (Frame Check Sequence (FCS)). Es una Comprobación de Redundancia de Ciclo (Cycle Redundancy Check (CRC)), computada desde la dirección de la casa al campo/zona de datos. El receptor computa el CRC del marco recibido y lo compara al valor de $\mathrm{CRC}$ recibido. Cualquier diferencia entre los dos valores indica que ha ocurrido un error durante la transmisión. En tal caso, el receptor desecha el marco. 
Para garantizar la transmisión en la línea, el EHS está provisto de un Corrector Avanzado de Errores (Forward Error Correction (FEC)). Un código FEC de 6 bites se añade a cada byte desde la dirección/código del hogar hasta el límite del cuadro. De esta forma cada byte se codifica con un valor de 14 bites. El método FTC tiene capacidad para corregir hasta tres bites consecutivos. Este método sirve para corregir errores de transmisión debidos al ruido que el manejo de los conmutadores de energía eléctrica genera. Cuando la señal es muy ruidosa, los errores se detectan a través de la secuencia de comprobación del marco.

\section{UN EQUIPO COMPLETO DE SOFTWARE Y HARDWARE}

Conectar un equipo a la red EHS requiere varios componentes. Para la comunicación de energía eléctrica, se establece la conexión con un módem de línea SGSThomson ST7537HS1 y su interface de línea. Un microcontrolador se encarga del protocolo EHS y de su aplicación. La aportación de energía eléctrica genera el voltaje suficiente para alimentar tanto el nodo de comunicación como el de aplicación.

\subsection{Productos software}

Para desarrollar una aplicación basada en el protocolo EHS, se ha escrito una biblioteca de programas software (HSLib) para la familia del microcontrolador SGSTHOMSON ST9. Trialog [3] genera la biblioteca software del protocolo asociada a un interface de Programas de Aplicación (Application Programming Interface (API)).

El API mantiene independiente la escritura software de la aplicación de la especificación y del protocolo EHS. Facilita la programación de una aplicación y la solución de imprevistos. Usando el API, la aplicación consiste en dos procedimientos de aplicación llamados State Machines. La transmisión y la recepción funcionan a través de memoria intermedia compartida entre aplicación y protocolo. La API provee de un programador con un juego completo de circuitos primitivos para el manejo de la memoria intermedia, los imprevistos, y la red.

Una aplicación escrita con API consite en dos procedimientos de aplicación llamados State Machines, a los que se recurre reiteradamente. La activación de las State Machines está programada por un módulo específico(HSLib Kernel) cuando ocurre algo relevante. Al activarla, la State Machine transmite el evento, procesa los que están en espera y se va. Cuando llegan nuevos incidentes, la State Machine se reactiva. La primera State Machine contiene los elementos rutinarios de programación de la aplicación, tales como el procedimiento de control y gestion I/O. La segunda State Machine se encarga del envío y recepción de órdenes, y se ocupa de servicios de gestión de red. Por ejemplo, el envío de un marco EHS desde la State Machine hasta un nodo remoto se hace por el procedimiento kernel, que estimula la llamada a las capas/niveles del protocolo HSLib para procesar la comunicación.

Para facilitar el desarrollo de un EHS basado en una aplicación, Trialog proporciona, además, otros productos software. HSTracer es un espía que reproduce un análisis de programa en tiempo real, en PC, de marcos y variables de aplicaciones EHS y que utiliza el software HSLib. HSWinLib es la versión Windows de la EHS Lib. Éste suministra la forma de conectar un PC a la red EHS. HSSimul es un software simulador de la red y las aplicaciones EHS.

\subsection{Productos hardware}

\subsubsection{Modem ST7537HSI de alimentador eléctrico}

En el EHS, los aparatos domésticos pueden intercambiar información transmitiendo datos en la red doméstica principal. El resultado es que no es necesario instalar cables suplementarios de control y se pueden conectar los aparatos simplemente utilizando el enchufe de pared más cercano.

Se conectan los electrodomésticos a la línea principal (mains) utilizando el Módem IC de la línea eléctrica de SGS THOMSON ST7537HS1. Éste envía y recibe datos a 2.400 bites por segundo (bps) en modo half duplex utilizando la frecuencia de recorrido de $132,45 \mathrm{kHz}$, que cumple con la Norma europea CENELEC EN 50065-1 y la reglamentación US FCC. La modulación de frecuencia FrequencyShift Keying (FSK) consigue una trasmisión enérgica en un entorno eléctrico muy ruidoso a un precio asequible para grandes consumidores y para mercados industriales.

El interface de la línea que conecta el equipo a los mains sólo necesita unos pocos componentes standard de exterior. El interface del módem al microcontrolador ST9 se hace con una conexión de cable de 7 . Se da la señal reset y la frecuencia master clock al microprocesador, reduciendo así el coste de la aplicación. Un imput watchdog protege el funcionamiento.

\subsubsection{Aportaciones del switch mode power}

Para ofrecer un diseño total de un punto de conexión de línea eléctrica, se propone la puesta en marcha del concepto power mode switch. Utilizando componentes comerciales de bajo coste, proporciona outputs de $5 \mathrm{~V} / 150 \mathrm{~mA}$ y $10 \mathrm{~V} / 300 \mathrm{~mA}$. Directamente conectados al conductor principal, se puede utilizar para suplir el propio punto de conexión y una aplicación sin necesidad de aislamiento. El espectro ruido se ha estudiado demostrando que los armónicos (movimiento de la onda) permenecen dentro de los límites establecidos por la norma CENELEC 50065-1. 
34

Informes de la Construcción, Vol. $50 \mathrm{n}^{\circ}$ 459, enero/febrero 1999

\subsubsection{Par trenzado/Transreceptor Coaxil}

La especificación EHS prevé un dispositivo de comunicación por medio de un par trenzado TP1 o un cable coaxil que utilice una codificación Alternate Mask Inversion (AMI) con una velocidad de datos de 9600 bps. A tal fin, se ha especificado un circuito integrado de transreceptor para hacer interface directamente entre un UART comercial a un par trenzado de tipo 1 o a un cable coaxil.

E1 TP1 actúa sobre la conversión de la señalización de datos entre la Grabación el Sin Vuelta a Cero (Non Return to Zero (NRZ)) y la codificación AMI. Comprueba el acceso al enlace común, actuando sobre la fase de arbitraje, detección de problemas, espacios de tiempo entre los datos y la señal de reconocimiento local. El transreceptor TP1 realiza directamente la interface del microcontrolador en chip Interface de Comunicación Serial (Serial Communication Interface (SCI)) vía unión con cable de 3 . También suministra reset/reajuste y señales master clock al microcontrolador. Se conecta directamente a las líneas de par trenzado.

\subsubsection{Microcontrolador ST9}

El software del protocolo EHS tiene acceso al microcontrolador ST9. La familia del microcontrolador ST9 se basa en una arquitectura modular de 8/19 bites que incluye un núcleo con un archivador de registros, un Interface Periférico Serial (Seria 1 Peripheral Interface) y un monitor de vigilancia (Watchdog). Varios microcontroladores están a disposición en los diferentes periféricos, tales como un Cronómetro Multiforma (Multiform Timer (MFT)), Interface de Comunicación Serial (Serial Communication Interface (SCI)), convertidor $\mathrm{A} / \mathrm{D}(\mathrm{A} / \mathrm{D})$ y varios tipos de memoria y tamaños, tales como RAM; EPROM; ROM y EEPROM todos ellos encajados dentro de una misma matriz.

Hoy, el protocolo del software EHS está incluido en un microcontrolador ST90E40 con dos cronómetros multiforma, un Interface de Comunicación Serial, un convertidor A/D, 16 kbytes de Eprom, 256 bytes de RAM, 512 bytes de EEProm. Este microcontrolador es lo bastante potente como para trabajar con el protocolo de comunicación y la aplicación del usuario. Para reducir el coste del nodo comunicación, es posible albergar la biblioteca EHS en un microcontrolador como el ST7.

\section{CONCLUSIÓN}

Los Sistemas Hogar Europeo han dado ya por terminadas las actividades de investigación y están entrando en la fase de industrialización. En EHSA se puede encontrar y solici- tar la especificación EHS. Para desarrollar los productos de automatización del hogar basados en EHS, existe disponibilidad de bloques constitutivos como el Modem Line de Energía Electrica ST7537HS1, el software del protocolo de comunicación e instrumento de comprobación de conformidad al EHS. Basándose en los bloques constitutivos, las compañías más importantes interesadas en las diferentes áreas de mercado, han iniciado ya el desarrollo de estos productos. Además, el proceso de normalización internacional de especificación y actividades afines a la inter-operatividad de aplicación están en marcha, y garantizarán una amplia aceptación de los EHS.

\section{BIBLIOGRAFÍA}

(1) ACERLI 4, rue René RAZEL, Saclay, F-91892 ORSAY Cedex. Tel.: + 33169351580 .

(2) FRAUNHOFER Institute Fraunhoferstr. 1, D-/6131 KARLSRUHE. Tel.: + 49726091372.

(3) TRIALOG 25, rue du Gal FOY 75008 PARIS. Tel.: +33 144 706108 .

(4) EHS specification R1.2 from EHSA, Excelsiorlaan 11, B1930 Zaventem, Belgium. Tel.: +32 7253232 .

(5) SGS-THOMSON Power line modem and application data book.

(6) Application note: A plug \& play demonstrator of the EHS network: EHSNODE.

(7) Application note AN655/0394: Power line modem application.

(8) Application note AN655/0394: 4.5W Flyback concerter for power line modem applications.

(9) SGS-THOMSON ST9 data books.

(10) Application note AN392/0992: Microntroller and triacs on the $110 / 240 \mathrm{~V}$ mains.

(11) Application note AN535/0894: Power line modem application remote control using ST7537 \& ST6.

(12) Application note AN430/0394: Synchronous PLM communication with ST9 multifunction timer.

(13) HSlib V1.03 manual Trialog.

(14) The EHS Network book. Edited by Trialog. 